\title{
L’accident nucléaire de Fukushima: enjeux et contestations autour de la définition du zonage
}

\section{Fassert ${ }^{*}$}

IRSN, Laboratoire de Recherche en Sciences Humaines et Sociales, Service Homme Organisation Technologie (SHOT), Pôle Sûreté Nucléaire, IRSN BP 17, 92262 Fontenay-aux-Roses Cedex, France.

Reçu le 16 janvier 2020 / Accepté le 10 février 2020

\begin{abstract}
Résumé - L'accident de la centrale nucléaire de Fukushima en 2011 a entraîné une crise de confiance des citoyens envers les autorités japonaises, et l'émergence d'une contre-expertise associative qui a questionné la légitimité des décisions prises. Dans cet article, nous nous centrons plus particulièrement sur la question du zonage comme outil de gestion des territoires contaminés (choix d'évacuation, seuil radiologique retenu, ...) et sur les critiques qui ont émergé dans l'espace public, en montrant ainsi certains enjeux démocratiques et éthiques de la politique post-accidentelle. Les analyses présentées résultent en grande partie des 120 entretiens effectués avec des habitants dans le cadre du projet SHINRAI entre 2014 et 2018.
\end{abstract}

Mots clés : Fukushima / territoires / contamination radiologique / zone / expertise / confiance

\begin{abstract}
The Fukushima Nuclear accident: stakes and disputes for the definition of zoning. The Fukushima Daïchi nuclear accident in 2011 raised a crisis of the trust of citizens towards the Japanese Authorities and the emergence of a contra-expertise of associations which have questioned the legitimacy of the decisions. In this article, we address more particularly the zoning as a tool of management of contaminated territories (choice of evacuation, choice of radiological threshold...) and the criticisms that emerged in the public domain and thus we highlight some democratic and ethical issues of the postaccidental policy. The analyzes presented here result for a large part from the 120 interviews of inhabitants during the SHINRAI project between 2014 and 2018.
\end{abstract}

Keywords: Fukushima / territories / radiological contamination / zone / expertise / trustworthiness

\section{Introduction}

Près de 10 ans après sa survenue, l'accident nucléaire ayant affecté la centrale de Fukushima le 11 mars 2011 laisse une trace profonde et durable dans la société japonaise. Il s'agit d'un accident majeur ayant entraîné une importante contamination terrestre et maritime. La gestion de l'accident nucléaire au Japon a reposé sur un ensemble de mesures de protection des populations (mises à l'abri dans l'immédiat post-accident, puis évacuations, mesures de surveillance sanitaire, ...) prises par les autorités en s'appuyant sur les recommandations édictées par la CIPR (Commission Internationale de Radioprotection) (ICRP, 2009) ou l'AIEA (Agence Internationale de l'Énergie Atomique) (IAEA, 2009) essentiellement.

La politique gouvernementale mise en place par différents acteurs au Japon [le MEXT (ministère de l'Éducation, de la Culture, des Sports, des Sciences et de la Technologie), la

\footnotetext{
*Auteur de correspondance : christine.fassert@irsn.fr
}

Nuclear Regulation Authority (NRA), ...] a reposé en premier lieu sur l'établissement de zones: zones évacuées et décontaminées avec ensuite «levée d'ordre d'évacuation», zones « difficiles pour le retour» dans lesquelles la contamination radiologique reste élevée, et zones restant interdites. D'autres aspects majeurs de la politique post-accidentelle, notamment ceux liés à la surveillance sanitaire des populations (dépistage des cancers de la thyroïde chez les enfants par exemple), font partie du paysage global de la situation.

Cet article examine plus particulièrement la question du zonage (définition de zones évacuées durablement, ou temporairement) comme instrument de gestion de la population vis-à-vis de la contamination radiologique. L'analyse porte essentiellement sur un élément devenu emblématique : le choix du seuil de $20 \mathrm{mSv}$ retenu pour la définition de zones d'évacuation temporaire, qui a entraîné une obligation, mais également un droit à l'évacuation assorti de mesures de compensations financières. Il s'agit d'un droit dont ont été par conséquent exclues les populations habitant des territoires dont la contamination radiologique n'atteint pas ce seuil. 
Les réflexions présentées ici résultent en grande partie des analyses des 120 entretiens effectués avec des habitants de Watari (ville de Fukushima), Nahara et Kawauchi dans le cadre du projet SHINRAI entre 2014 et 2018 mené conjointement par l'Institut de radioprotection et de sûreté nucléaire (IRSN), Sciences Po Paris et l'Université de technologie de Tokyo (Fassert et Hasegawa, 2019). Le projet SHINRAI (mot japonais qui désigne la confiance), engagé en 2014, avait notamment pour but de comprendre finement les mécanismes de perte rapide de la confiance des citoyens envers les autorités et leurs experts et d'analyser les modalités de l'émergence de nouveaux experts citoyens, ou «contre-experts». Les huit missions de 2 à 3 semaines du projet SHINRAI ont permis de rencontrer, parfois à plusieurs reprises, des habitants de retour dans leur ville d'origine après les levées d'ordre d'évacuation par le gouvernement, mais aussi des habitants qui ont été évacués dans des lieux parfois éloignés de Fukushima (Kyoto, Nagasaki...). Elles ont permis également de rencontrer des représentants des autorités nationales et locales, et enfin des associations et des «contre-experts», notamment du monde « anti-nucléaire », qui ont joué un rôle important d'information et de soutien auprès des populations.

Cet article s'intéresse particulièrement à la contestation du seuil de zonage de $20 \mathrm{mSv}$ par un ensemble d'acteurs, au Japon et au niveau international, qui ont dénoncé un seuil qu'ils considéraient trop élevé, ou bien qui ont jugé qu'une situation accidentelle ne devait pas remettre en question la norme des $1 \mathrm{mSv} /$ an établie par la CIPR pour le public, en dehors des situations accidentelles, mais que le Japon n'a pas transposé dans son corpus réglementaire.

Le relèvement du seuil de 1 à de $20 \mathrm{mSv} /$ an a été décidé par le gouvernement japonais à partir du schéma établi par des recommandations de la CIPR (Shirabe et al., 2015) et justifié en s'appuyant sur des arguments scientifiques controversés, qui sont étroitement liés à l'histoire même de la radioprotection (Boudia, 2008). À peine annoncé, il a été l'objet d'une large contestation, qui a cristallisé les nombreuses critiques apportées à la politique de gestion post-accidentelle mise en place par les autorités. En outre, la question du seuil nous intéresse particulièrement car elle a été soulevée à deux reprises: au moment du zonage pour décider qui devait/pouvait évacuer (l'évacuation obligatoire fait l'objet de compensations financières pour les victimes), et au moment de la levée des ordres d'évacuation, décidée au cas par cas pour chaque ville évacuée. Dans ces deux cas, le choix d'un seuil, par le gouvernement, est contesté

Cette contestation constitue un objet de recherche à part entière, car elle permet d'examiner de nombreuses questions liant les domaines de la science, de l'expertise et des décisions politiques. La sociologie de la confiance (Quéré, 2006) permet de montrer comment s'articulent, dans cette situation, la contestation du seuil et la perte de confiance de certains citoyens envers les autorités. L'analyse de cette contestation a aussi été réalisée dans le projet de recherche SHINRAI, grâce à l'examen de rapports réalisés par les principaux acteurs associatifs en France (rapport Greenpeace, 2017, Rapport ACRO (Boilley, 2016), au Japon (rapport du Citizens' Commission on Nuclear Energy -; CCNE, 2015) ou encore à l'international (IPPNW/PSR, 2016). Du côté institutionnel, le rapport du Rapporteur Spécial de l'ONU a été également examiné. Cette analyse a été complétée par des entretiens menés avec les principaux acteurs, scientifiques et associatifs, de cette contestation. Seuls quelques éléments de cette analyse sont présentés ici, en insistant sur un des effets majeurs de cette contestation: la re-politisation des questions et des problèmes liés à l'accident nucléaire, que le gouvernement japonais avait essentiellement abordés sur la base d'arguments strictement scientifiques.

\section{Le zonage: historique et mise en œuvre après l'accident de Fukushima}

\subsection{Le cadre historique}

La politique de zonage établie par le gouvernement japonais s'inscrit dans un cadre de référence établi par les deux grandes institutions internationales que sont l'AIEA et la CIPR. Topçu rappelle quelques éléments historiques du «dispositif de zonage», en montrant comment il s'est imposé dans les années 1960 comme une modalité essentielle de gestion de l'accident nucléaire (Topçu, 2016). L'auteur rappelle comment, dès les années 50 , les conséquences d'un accident nucléaire sur les territoires ont été estimées suffisamment importantes pour justifier que des mesures soient prises a priori. Ainsi, un comité d'experts de l'Atomic Energy Commission (AEC), aux États Unis, proposa même d'isoler les sites nucléaires, avec un périmètre d'exclusion proportionnel à la puissance du réacteur : cela entraînait, par exemple, une exclusion de $30 \mathrm{~km}$ pour un réacteur d'une puissance de $1000 \mathrm{MW} / \mathrm{h}$. Cette solution ne fut pas retenue, car les industriels y virent une source d'angoisse pour les populations voisines, et de possible rejet de cette industrie. En 1956, l'AEC retient donc une autre solution: la définition de zones d'évacuation autour des centrales, en cas d'accident. Le rapport dit « Wash $740 »$ (USAEC, 1957) établit un dispositif en 5 zones (mise à l'abri, évacuation temporaire, définitive, et les autres...) qui sera plus tard appliqué dans ses grandes lignes lors des accidents de Tchernobyl et de Fukushima. Pour Topçu, ce dispositif de zonage a pour principal objectif d'affirmer que le problème est ou va être localisé, « encerclé, et que par conséquent la menace était sous contrôle». Ce dispositif introduit également des nuances entre différentes catégories de populations, qui accéderont à des droits différents en termes, notamment, de compensation. La double dimension, symbolique et économique, du zonage comme dispositif politique est bien montrée ici. Symbolique, car: «des zones d'évacuation trop étendues dans l'espace comme dans le temps étaient et restent à éviter, aux yeux des nucléaristes, parce qu'elles rendent trop visible le caractère inacceptable des risques encourus » (Topçu, 2016), et économique car l'évacuation des populations est accompagnée de mesures de compensions financières qui seront essentiellement supportées par l'Etat, étant donné le régime de responsabilité restreint auquel est soumise l'industrie nucléaire (Topçu, 2014).

\subsection{Le zonage comme outil d'administration des territoires contaminés}

Du 12 mars à fin avril 2011, les premières mesures sont prises dans une situation de crise dramatique, et dans un contexte de grande incertitude sur la cinétique de l'accident de Fukushima et les rejets radiologiques. Des ordres successifs d'évacuation incluant un périmètre grandissant autour de la 


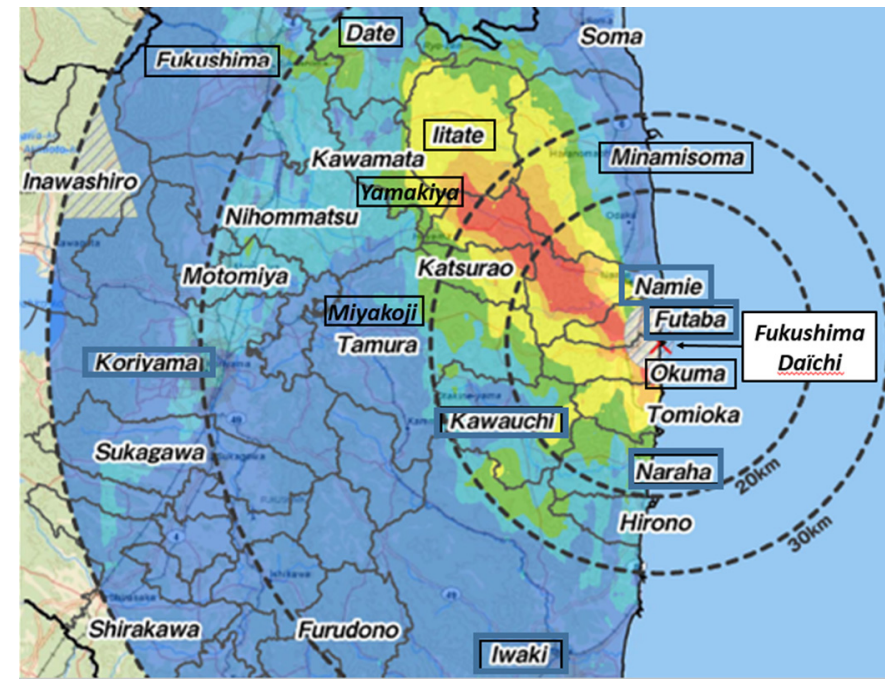

Fig 1. Cartographie des dépôts radioactifs au sol en mai 2011 dans la région de Fukushima et localisation des villes mentionnées dans cet article. Source U.S. Department of Energy.

centrale, ainsi que des ordres de confinement de la population, sont émis. Fin avril 2011, avec l'établissement plus précis de cartes de contamination radiologique, une zone d'évacuation obligatoire est établie au-dessus des $20 \mathrm{mSv} / \mathrm{an}$, suivie d'ordres d'évacuation un mois plus tard pour des «points spécifiques recommandés pour l'évacuation ». Les villes de Kawauchi et de Naraha sont dans cette zone d'évacuation obligatoire. Une grande partie des habitants de Naraha est évacuée à Iwaki (Fig. 1). De nombreux habitants de Kawauchi ont été quant à eux déplacés à Koriyama. Une zone de «retour difficile» est également définie dans laquelle la contamination est au-dessus de ces normes: les villes de Futaba, Okuma, Namie en font partie. Pour le premier type de zone, des opérations de décontamination intenses ont été lancées, et le gouvernement a affiché clairement dès le départ une volonté de retour rapide des populations après les opérations de décontamination.

\subsection{Les décisions gouvernementales autour du seuil et du zonage}

Le zonage va établir et réifier une territorialisation d'un risque pourtant éminemment difficile à circonscrire spatialement et temporellement: les limites établissent l'obligation d'évacuer pour les populations concernées, mais aussi des droits à compensation financière, qui vont être mis en place par l'Agence pour la Reconstruction, établie sous l'égide du METI (ministère de l'Économie, du Commerce et de l'Industrie). Le zonage est aussi, dans le même temps, un dispositif excluant de ces droits des personnes qui peuvent être dans une situation radiologique pourtant très proche. Dans « The land of hope», le cinéaste Sion Sono montre comment deux familles voisines et amies, sont brutalement séparées à la suite d'un accident nucléaire, avec une frontière tracée entre leurs maisons, qui établit la limite fixée entre une zone interdite et évacuée et une zone qui resterait habitable.

Il est difficile de retracer clairement les critères qui ont prévalu dans le choix d'un seuil de $20 \mathrm{mSv} / \mathrm{an}$ au Japon. Les autorités n'ont pas évoqué d'arbitrages dans les décisions prises, elles ont communiqué en justifiant ce seuil sur la base d'arguments scientifiques insistant sur la non dangerosité des faibles doses, ou même sur le fait qu'il n'y avait pas de dangers prouvés pour des radiations en dessous d'un seuil de $100 \mathrm{mSv}$. Elles ont aussi affirmé avoir choisi la limite basse des valeurs de référence de la CIPR pour les situations d'urgence radiologique $(20-100 \mathrm{mSv})$. En vérité, lorsque ce seuil est fixé en avril 2011, il ne s'agit plus d'une situation d'urgence radiologique: les personnes évacuées avaient un mois pour partir. Le Japon était déjà dans ce que la CIPR qualifie de «situation existante» pour laquelle les valeurs de référence doivent être choisies entre 1 et $20 \mathrm{mSv} / \mathrm{an}$ et diminuer avec le temps (Fassert et Hasegawa, 2019).

Cependant, le choix d'un seuil de $20 \mathrm{mSv} / \mathrm{an}$ ne repose pas uniquement sur la prise en compte des effets des rayonnements ionisants sur la santé. Des outils de simulation permettent de rendre visibles les conséquences d'un seuil d'évacuation pour un territoire donné. L'IRSN a ainsi calculé que le choix d'un seuil à $10 \mathrm{mSv}$ au Japon pour la définition de zones aurait eu des conséquences importantes: une partie de la ville de Fukushima (à $50 \mathrm{~km}$ de la centrale), aurait été évacuée, entraînant l'évacuation de 70000 personnes supplémentaires, ce qui aurait engendré un coût important ainsi qu'un message symbolique fort sur les conséquences de l'accident. De plus, les autorités ont argumenté que les évacuations et les conditions de vie des évacués dans les lieux d'hébergement temporaire étaient également à l'origine d'effets non négligeables sur la santé. Un premier ensemble de critiques relevées dans nos entretiens porte donc sur le fait que ces arbitrages impliquant d'autres dimensions que le critère sanitaire n'ont pas été mentionnés par le gouvernement, et encore moins discutés et publiquement débattus. Le Professeur Yamauchi (Fassert et Hasegawa, 2019), par exemple, estime que le seuil de $20 \mathrm{mSv} / \mathrm{an}$ a été choisi essentiellement pour éviter d'évacuer la région de Naka Dori et ses grandes villes: le tracé permettait de concentrer les zones d'évacuation sur la côte, en évitant d'évacuer les grandes villes du centre de la préfecture. «Fukushima est la capitale, c'était symbolique, on ne pouvait pas l'évacuersans reconnaître l'ampleur des conséquences de l'accident nucléaire».

Ces calculs et ces arbitrages n'ont pas fait l'objet de débats publics, et la question économique est restée très largement soustraite à l'examen politique, et à sa critique. Les arguments présentés par les autorités ont été strictement radiologiques, se focalisant sur le caractère «non dangereux» du seuil de $20 \mathrm{mSv} / \mathrm{an}$. La question de l'arbitrage économique (ou socioéconomique) réalisé n'a pourtant pas été largement abordée dans les contestations initiales, qui se sont focalisées pour l'essentiel sur le caractère trop peu conservatif du seuil, notamment pour les enfants.

\subsection{Une communication « rassurante »}

Dans les semaines qui ont suivi l'accident, et alors même que certaines zones n'étaient pas encore évacuées, le porteparole du gouvernement, Yukio Edano a répété à plusieurs reprises: "The current level of radiation has no immediate effect on your health ». Un membre du Liberal Democratic Party lui reprochera ensuite d'avoir répété cette phrase à de nombreuses reprises. Cette phrase a été très diffusée par les 
media, qui reprenaient en outre des données de comparaison avec les expositions médicales (Yuasa, 2015). Le rapport de la commission parlementaire (rapport NAIIC) juge que cette phrase a «donné au public un sentiment erroné de sécurité» (Boilley et Josset, 2016).

Lors des entretiens que nous avons menés avec des habitants de la Préfecture de Fukushima, plusieurs personnes ont évoqué cette fameuse déclaration du porte-parole Edano, en soulignant que c'est à partir de ce moment-là qu'elles ont perdu la confiance envers les autorités de leur pays. (La question posée était précisément : à quel moment précis avezvous perdu confiance envers les autorités ?). En effet, la formulation même utilisée par le porte-parole du gouvernement apparaît comme se voulant « rassurante» tout en évitant d'être fausse. Le «no immediate effects» a été perçu comme une façon de se prémunir des critiques: le propos n'est pas scientifiquement faux, puisque les effets sanitaires sont effectivement souvent tardifs mais la formulation contient un élément rassurant qui apparaît comme relevant d'une forme d'hypocrisie, en tout cas de volonté de minimiser l'impact sanitaire de l'exposition aux radiations ionisantes.

Les gouvernements successifs ont adopté un discours monolithique sur le risque radiologique, insistant sur l'absence de preuves de dangers en dessous du seuil de $100 \mathrm{mSv}$ et en ne mentionnant pas l'existence d'une controverse déjà ancienne sur les dangers des faibles doses de radiations ionisantes. La contestation de ce seuil par les acteurs institutionnels, associatifs, scientifiques fera au contraire resurgir l'existence de cette controverse dans l'espace public, la déconfinant des cercles étroits des spécialistes en radioprotection. Ces critiques s'appuient sur une vision beaucoup plus conservatoire des risques, reposant sur le modèle dit «linéaire sans seuil». (Boudia, 2014). Ce modèle stipule qu'il n'est pas possible d'établir un seuil en dessous duquel on serait assuré de l'innocuité des radiations ionisantes, et ce depuis les travaux de l'Académie Nationale des Sciences aux États Unis qui fut l'une des premières à produire un rapport public sur les effets des essais atmosphériques en 1956 (BEIR VII, 2006).

Le déni de la controverse sur les faibles doses, et plus généralement le déni des incertitudes concernant la question de la dangerosité des radiations ionisantes, associé à une communication unilatérale sur la non-dangerosité des radiations ionisantes a joué un rôle important dans la perte de confiance des japonais. Dans ce contexte, la figure du «goyo gakusha», scientifique défendant le seuil choisi, et plus généralement l'innocuité des faibles doses de radioactivité, a émergé. L'expression dénonce le scientifique ou l'expert inféodé aux intérêts du gouvernement japonais et porteur d'un discours soutenant avant tout les avis scientifiques en accord avec la politique gouvernementale. Cette expression sera très souvent utilisée pour dénoncer dans le contexte postaccidentel, un pouvoir scientifique étroitement lié aux intérêts gouvernementaux.

\section{Une contestation multiforme}

\subsection{La contestation interne}

Dans ce contexte, une contestation de la politique postaccidentelle au Japon s'est développée rapidement dans les mois qui ont suivi la catastrophe.
Très rapidement, au sein même du gouvernement, des personnes vont se désolidariser de la politique post-accidentelle menée par le gouvernement, et particulièrement de l'emblématique «seuil» retenu. Le conseiller spécial en radioprotection du Cabinet du Premier Ministre, le professeur Toshiso Kosako, un éminent chercheur de l'université de Tokyo, démissionne en larmes le 30 avril 2011: «Je ne peux pas accepter un tel seuil, appliqué aux bébés, enfants, et élèves des écoles primaires, pas seulement d'un point de vue universitaire, mais aussi en raison de mes valeurs humanistes (...) 'Si j'approuvais cette décision, je ne serais plus un chercheur. Je ne voudrais pas que mes enfants soient exposés à de telles radiations" $"$.

Le rapport commandé par la commission parlementaire se montrera lui aussi critique sur la gestion post-accidentelle (NAIIC, 2012). Sur la question qui nous intéresse, le rapport rappelle l'absence de consensus sur la dangerosité des faibles doses, et insiste sur le «manque d'informations » données aux résidents et sur les incertitudes liées aux dangers des faibles doses : "While exposure levels are set as a threshold against acute radiation disorder, there is no widely accepted threshold for long-term radiation damage caused by low doses. The international consensus, however, is that the risk does increase in proportion to the dose».

\subsection{La contestation associative}

L'accident nucléaire a également eu pour conséquence, sans surprise, de raviver la contestation anti-nucléaire (Gaulène, 2016). Des manifestations mensuelles sont organisées à Tokyo les mois suivant la catastrophe, le mouvement se structure autour de Sayonara Genpatsu: Kenzaburo Ôe, prix Nobel de littérature est présent à la manifestation du 19 septembre 2011. Des mouvements se structurent: «Occuper Kasumigaseki (le quartier des ministères à Tokyo) », et une manifestation importante la «révolution des hortensias» le 29 juin 2012. Ces évènements, comme le regrette Keiko Courdy, sont cependant peu couverts par la presse (Courdy, 2013).

Ce monde comprend d'abord des ONG "établies" de longue date dans l'opposition à l'industrie nucléaire comme par exemple Green Action Japan ou Greenpeace. L'accident est pour ces associations l'occasion de dénoncer les risques liés à l'industrie nucléaire, mais aussi la politique gouvernementale de gestion de la catastrophe. Ces associations mènent des actions d'aide aux populations, et de protestation. En août 2012, une requête est présentée au Haut-commissariat aux droits de l'homme des Nations Unies, par un ensemble d'associations. Elle est intitulée: «Violations des droits des enfants de Fukushima ${ }^{1}$. Elle réclame la «rétractation» du relèvement du seuil de $20 \mathrm{mSv} /$ an pour les enfants, et que « soit révélés les noms des experts qui ont établi que le seuil de $20 \mathrm{mSv} / \mathrm{an}$ était correct pour les enfants...».

\footnotetext{
${ }^{1}$ Requête présentée par: Green Action, Greenpeace Japan, Citizens' Nuclear Information Center, Citizens Against Fukushima Aging Nuclear Power Plants (Fukuro-no-Kai), Osaka Citizens Against the Mihama, Oi, and Takahama Nuclear Power Plants (Mihama-no-Kai), Friends of the Earth Japan.
} 
Le Citizen's Nuclear Information Center (CNIC), créé en 1975 par Jinzaburo Takagi (fondateur de la Takagi School) prend également publiquement position. Il se fait entendre dans les jours qui suivent l'accident grâce au club des correspondants de presse étrangers, (Gaulène, 2016), et développe une contre-expertise sur de nombreux sujets liés à l'analyse de l'accident nucléaire et à sa gestion. L'opposition au seuil de $20 \mathrm{mSv} / \mathrm{an}$ est un élément central de la critique adressée.

À côté des opposants de longue date, de nouvelles structures naissent dans le contexte de l'accident nucléaire. Le CRMS (Citizen Radiation Monitoring System) est fondé par Wataru Iwata, un compositeur de musique, qui met en place un réseau de stations de surveillance radiologique, ainsi que des appareils de mesure de la contamination de la nourriture. Il reçoit de l'aide de la Commission de recherche et d'information indépendantes sur la radioactivité (CRIIRAD) pour ses actions de mesure et d'information. Chikurin-sha est une association fondée par des associations environnementalistes, dont Fukuroo no kai, une association anti-nucléaire, qui existait avant l'accident nucléaire de Fukushima; Chikurinsha propose des mesures de la contamination interne grâce à l'acquisition d'un appareil au Germanium. Cet appareil est financé notamment par le Conseil General d'Ile de France, et un support technique et scientifique est assuré par l'Association pour le Contrôle de la Radioactivité dans l'Ouest (Fassert et Hasegawa, 2019). Saving Children from Fukushima est fondée par des parents inquiets, et propose des actions entièrement tournées vers les enfants : organisation d'un réseau de mesures de la radiation ambiante se focalisant sur les chemins des écoliers, les mesures dans les parcs, les écoles, et aide aux familles qui veulent évacuer alors qu'elles sont en dehors des zones d'évacuation définies par les autorités (autoévacués).

Une partie des associations vont articuler leur critique de la politique post-accidentelle à une critique plus large de l'énergie nucléaire. La dénonciation des conséquences de l'accident pour les populations réactive la critique envers une industrie nucléaire qui s'était érigée sur la base de ce que ces associations appellent le «safety myth». D'autres acteurs se démarquent volontairement d'une posture anti-nucléaire. Ainsi, une association fondée dans les mois qui ont suivi l'accident, SAFECAST, se focalise sur la mesure de la contamination radiologique en proposant la mise en ligne des données mesurées par les citoyens, à l'aide d'un compteur Geiger qu'ils fabriquent eux-mêmes dans un «fab-lab». «We are neither pro nor anti nuclear, we are pro data » déclarent-ils.

\subsection{La contestation internationale}

"An accident somewhere is an accident everywhere» (Blix, 2005) : cette phrase peut être interprétée de différentes manières. Elle peut notamment attirer l'attention sur le fait qu'une politique post-accidentelle dans un pays, après un accident nucléaire, sera évaluée, non seulement par les citoyens directement impliqués, victimes de l'accident, mais également au niveau international, où elle fera l'objet de commentaires, critiques, dénonciations, qui ré-activeront des questions récurrentes, faisant le lien entre, non seulement les accidents nucléaires civils, mais aussi entre accidents nucléaires et usages militaires de l'atome. Ainsi, après l'accident de Fukushima, la communauté internationale des scientifiques structurée autour de l'abolition de l'arme nucléaire va intervenir à plusieurs reprises.

Ainsi, en mai 2012, l'International Physicians for Prevention of Nuclear Wars (IPPNW) écrit une lettre officielle au MEXT à propos du seuil de $20 \mathrm{mSv} / \mathrm{an}$ (Yuasa, 2013). Cette lettre exprimait «a deep concern » à propos du seuil, et évoquait notamment les travaux du BEIR VII de l'US National Academy of sciences. La lettre insistait également sur l'existence d'une plus grande sensibilité des enfants aux radiations ionisantes. Yuasa s'intéresse cependant à une absence importante : les signataires de cette lettre n'incluent pas la branche japonaise de l'IPPNW (la Japanese Physicians for Prevention of Nuclear Wars). Le président de la JPPNW s'est expliqué sur son refus de signer ainsi : «I can understand that this letter represents justified concerns, including that children exposure to the radiation should be aimed for zero. However, I did not add my signature because I think that IPPNW should not engage in anti-government activities ». En août 2012, la $20^{\mathrm{e}}$ conférence de l'IPPNW s'est tenue à Hiroshima et les discussions qui s'y sont tenues ont révélé de profondes dissensions. En effet, les conclusions de la session organisée par la branche Japonaise sur l'accident de Fukushima et ses conclusions sont considérées comme trop indulgentes à l'égard de la politique gouvernementale post-accidentelle. Ce différend qui oppose l'IPPNW et sa branche japonaise s'inscrit dans un cadre plus large: la branche Japonaise de l'association n'a jamais condamné l'usage civil du nucléaire, contrairement à la INPPW, et ce y compris après l'accident de Fukushima. Il faut noter qu'au Japon, contrairement à d'autres pays, le combat contre le nucléaire militaire n'a pas épousé celui contre le nucléaire civil, un grand nombre de survivants des bombardements ayant adhéré au discours de «Atoms for Peace» (Jobin, 2016).

\subsection{La contestation issue du monde scientifique et académique}

Plusieurs chercheurs vont intervenir sur la scène publique pour dénoncer le seuil retenu. Certains de ces scientifiques ont déjà une longue histoire dans l'opposition anti-nucléaire, et l'accident va déclencher ou développer leur critique quant à la politique gouvernementale. Dans ce contexte, la critique du seuil n'est qu'un élément d'une critique plus large. Ainsi, le professeur Makino de l'université de Tokyo, un chercheur en Astrophysique, et le professeur Koide, du Kyoto University Research Reactor Institute (KURRI), opposant de longue date à la poursuite du programme nucléaire japonais deviennent des contre experts majeurs sur la scène publique. Tous deux expriment leur désaccord avec le seuil retenu.

Un autre aspect de la critique consiste à comparer le seuil choisi par le gouvernement japonais au seuil retenu pour Tchernobyl $(5 \mathrm{mSv})$. Le professeur Yukio Hayakawa, volcanologue de l'université de Gunma, édite une carte montrant les zones contaminées autour de Tchernobyl et de Fukushima. Cette carte devient une référence dans les mouvements civils, elle est placardée dans presque tous les centres d'aide et d'entraide (Sugita et Augendre, 2012). 
Hisako Sakiyama, professeure de médecine et ancienne directrice de recherche au NIRS (National Institute of Radiological Sciences) avait alerté lors de ses travaux de recherche réalisés bien avant l'accident nucléaire, sur les dangers des expositions médicales. Elle rejoint à sa retraite du NIRS la Takagi School for Alternative Scientists. Elle siège dans la Commission parlementaire dite NAIIC et elle critique sévèrement la minimisation du risque radiologique par certains des experts gouvernementaux, notamment à l'occasion de son audition. Elle tient à souligner le point suivant, lors d'un entretien récent : «En 2015 les arguments du gouvernement restent les mêmes que juste après l'accident. Pourtant, entre temps, il y a eu la publication INWORKS qui montre la validité du modèle linéaire sans seuil, et une importante étude australienne par Matthews, qui a établi de façon très claire un lien entre les expositions médicales et l'augmentation des cancers. On peut considérer qu'il n'y a plus de véritable controverse, au sens scientifique, sur les faibles doses. Même (les professeurs) Niwa et Yamashita le savent. Mais ils cherchent avant tout à rassurer la population et à justifier les décisions prises » (Fassert et Hasegawa, 2019).

Une autre critique est apportée dans l'espace public par le professeur Tatsuhiko Kodama, directeur d'un centre de recherche en radiologie à l'University of Tokyo, qui a été appelé comme témoin à la commission du travail et santé du parlement Japonais. À cette occasion, il critique violemment les politiciens pour leur absence de mesures de protection pour les enfants. Les images vidéo montrant sa colère et sa dénonciation de la politique gouvernementale, accusée de négligence envers les spécificités du risque radiologique pour les enfants vont largement circuler sur le web, et le Professeur Kodama va apparaître comme un scientifique attentif à la vulnérabilité des plus faibles. Lors d'un entretien il répond, à propos de nos questions sur ce qui lui apparaîtrait comme un seuil «juste» : "Il y a un seuil de $1 \mathrm{mSv}$ /an établi par la CIPR, pourquoi devrait-on choisir un autre seuil pour les situations accidentelles?»(Fassert et Hasegawa, 2019). Trois ans plus tard, lorsque le maire de Kawauchi doit constituer un «comité pour le retour dans les territoires évacués» il choisit de demander au professeur Kodama d'être un des experts de ce comité : «Je suis allé le voir à Tokyo, j'avais été ému de le voir protester contre ce seuil pour les enfants, cela m'a décidé à le choisir comme conseiller » (Fassert et Hasegawa, 2019).

Des intellectuels comme le Professeur Shimazono, spécialiste des religions, de Sophia University sont également intervenus (Fassert et Hasegawa, 2019). Shimazono fait partie du CCNE (Citizen commission on Nuclear Energy), et il est membre de l'académie des sciences du Japon. Durant le « 6th Citizen-scientist International Symposium on Radiation Protection », il préside la journée de clôture, durant laquelle est évoquée l'idée de formuler une conclusion rappelant que les mesures prises par les autorités devraient respecter le modèle LNT, car il s'agit du consensus le plus large existant dans la communauté scientifique.

\subsection{De la critique du seuil à la critique de la Radioprotection : le rapport du Rapporteur Spécial de l'ONU}

Un autre type de critique parvient de la sphère institutionnelle internationale. Anand Grover, rapporteur spécial, soumet le
27 mai 2013 un rapport au conseil des droits de l'homme des Nations Unies, après une mission d'enquête menée à la suite de l'accident de Fukushima (Grover, 2013).

Le rapport se montre très critique sur la politique globale du gouvernement japonais en réponse aux conséquences de l'accident nucléaire. Il relève des éléments très concrets qui ont entaché sérieusement la confiance des habitants envers l'État, notamment l'existence de «points chauds» mesurés près de stations de mesure installées par les autorités, stations qui ne les détectent pas, ce qui génère la méfiance des citoyens envers les mesures effectuées.

Une critique majeure concerne la communication du gouvernement au sujet des risques radiologiques. Cette communication induit chez les citoyens, selon le rapporteur, une compréhension erronée du risque radiologique, trop rassurante: "The supplementary reading and presentation materials mention that there is no clear evidence of excess risk of diseases, including cancer, when exposed for a short time to radiation levels of $100 \mathrm{mSv}$ and below. This gave the impression that doses below $100 \mathrm{mSv}$ are safe. Un autre point, et non des moindres, car repris par plusieurs associations et scientifiques qui en feront un argument d'ordre éthique à la portée émotionnelle indubitable, la communication gouvernementale omet de rappeler la sensibilité particulière des enfants aux radiations ionisantes: "The textbooks do not mention the increased vulnerability of children to the health effects of radiation. Such information may give children and parents a false sense of security, which may result in children's exposure to high levels of radiation $\gg$.

Le seuil de $20 \mathrm{mSv} / \mathrm{an}$ est par conséquent jugé tout simplement inapproprié aux regards des aspects réglementaires, légaux, scientifiques. Le rapport recommande en conclusion de revenir à la limite de $1 \mathrm{mSv}$ de dose additionnelle annuelle, ce qui implique, entre autres choses, de fournir une assistance pour l'évacuation de ceux qui souhaitent partir audelà de cette limite, et de se donner cet objectif pour la décontamination des territoires contaminés: "Comme les effets négatifs pour la santé sont possibles pour des faibles doses de radiation, il ne devrait être recommandé aux évacués de rentrer qu'à partir du moment où la dose de radiations a été réduite autant que possible et à un niveau inférieur à $1 \mathrm{mSv} / \mathrm{an}$. En attendant, le gouvernement devrait continuer à fournir un soutien financier et de quoi vivre à toutes les personnes évacuées de façon à leur permettre de décider volontairement de retourner dans leur foyer ou de rester évacuées » (Boilley, 2016). À la suite de cette analyse, Hasegawa (2015) montre que le cadre international des Internally Displaced Persons (IDPS) pourrait alors être appliqué en cas d'accident nucléaire : "Under no circumstances should IDPs be encouraged or compelled to return or relocate to areas where their life, safety, liberty or health would be at risk".

Cependant, un des éléments les plus radicaux de ce rapport est qu'il va bien au-delà de la critique du relèvement du seuil à $20 \mathrm{mSv} / \mathrm{an}$, et remet en question les fondements mêmes de la radioprotection, qui reposent sur le principe ALARA : As Low as Reasonably Achievable. Ce «reasonably» indique que des critères autres que sanitaires sont pris en compte. Ce que critique Anand Grover, en s'appuyant pour cela sur le « Droit à la santé»(Grover, 2013). Le rapporteur précise en effet que «les recommandations de la CIPR sont basées sur le principe d'optimisation et de justification, selon lesquelles toutes les 
actions du gouvernement doivent maximiser les bénéfices sur le détriment. Une telle analyse risque-bénéfice n'est pas en accord avec le cadre du droit à la santé, parce qu'elle donne la priorité aux intérêts collectifs sur les droits individuels. Le droit à la santé impose que chaque individu doit être protégé. De plus, de telles décisions, qui ont un impact à long terme sur la santé physique et mentale des populations, doivent être prises avec leur participation active, directe et effective ».

La critique adressée ici ouvre un nouveau type de contestation, plus radicale que les critiques qui se sont intéressées, par exemple, à la sensibilité particulière des enfants aux radiations ionisantes, puisqu'elle met en question la notion même du cadre ALARA, qui prend en compte le « reasonably », c'est à dire le fait de limiter la prise en compte de la santé à ce qui est « raisonnablement faisable». En outre, il montre l'apparition d'une «compétition entre légitimités » (Rosanvallon, 2008): les cadres du droit international à la santé apparaissent ici peu compatibles avec le cadre de la CIPR, ou encore les recommandations de l'AIEA, qui vont au contraire reposer en premier lieu sur des «analyses coûts bénéfices» permettant de mettre en perspective les risques sanitaires avec d'autres «risques», économiques et psycho sociaux. Ces modes de régulation dans le nucléaire ne sont pas spécifiques, ils peuvent au contraire être analysés comme liés à ce que Boudia et Jas (2014) nomment le développement d'un «monde toxique» où les systèmes de régulation, ayant « échoué à endiguer le développement de substances nocives, s'attachent à développer des formes subtiles d'acceptabilité des risques, toujours basées sur l'idée implicite que l'option de la suppression de ces substances » (ou leur évitement radical dans le cas de la contamination radioactive) n'est pas envisageable. Cependant, ces modes de régulation se heurtent à des droits internationaux, sans toutefois que cette «incompatibilité» apparaisse de manière manifeste dans l'espace public, en dehors, justement, du moment de crise constitué par l'accident nucléaire.

\section{Des réponses gouvernementales peu audibles}

\subsection{La communication comme solution}

Face à ces nombreuses critiques, le gouvernement a réagi essentiellement en insistant sur la nécessité de communiquer. Le paradigme général était que les décisions n'étaient pas comprises par la population, qu'il fallait mieux justifier leur rationalité, trouver les bonnes explications à donner. Un comité est créé : le comité pour la sécurité (anzen) et le sentiment de sécurité (anshin), dont le titre même entérine une vision très répandue : il existerait une vision «juste» des risques, mais les habitants de la préfecture de Fukushima souffriraient d'une vision exagérée des risques radiologiques, qu'il conviendrait de corriger. Une autre dimension de la communication porte sur la comparabilité du risque radiologique : «les faibles doses de radioactivité ne sont pas plus dangereuses que de ne pas manger 5 fruits et légumes par jour » explique un expert dans une réunion d'information. La commensurabilité et la comparabilité des risques semblent ne pas avoir de limites pour certains. À propos du cas de familles qui se séparent, à cause d'un désaccord dans le couple, lorsque des mères partent avec leurs enfants laissant leur mari dans la préfecture de
Fukushima, un responsable du comité «anshin/anzen» considère: "vivre dans un endroit légèrement radioactif est moins risqué que de vivre sans père».

De nombreuses actions de communication sont lancées, ainsi qu'un important programme d'éducation sur les radiations ionisantes, et par exemple, la nomination de personnes chargées d'expliquer au public les risques radiologiques dans les différents villages après le retour. À propos de la présence d'une jeune femme, cadre de santé, venue s'installer à Kawauchi, plusieurs habitants nous disent, lors d'entretiens : « elle est là pour nous rassurer ». Ils considèrent même qu'au-delà des actions de conseil qu'elle est amenée à réaliser auprès des habitants (ne pas consommer les produits de la forêt, ou en faire mesurer la radioactivité par exemple), sa présence même, en raison de son jeune âge, est utilisée comme une action de communication à part entière.

Il apparaît cependant qu'une fois la confiance perdue, ou très sérieusement entachée, le surcroît de communication est inefficace, puisque les informations sont données par une institution à laquelle les habitants ne font plus confiance. Comme l'a montré Wynne dans son étude des suites de Tchernobyl dans la région de Lake district (Angleterre), les fermiers ne font pas confiance aux experts au moment de la gestion des retombées radioactives de Tchernobyl parce qu'ils ont peu confiance, depuis l'accident de Sellafield en 1957, dans les mêmes experts et autorités qui avaient délibérément caché au moment de cet accident des informations cruciales sur la contamination générée par l'incendie (Wynne, 1992).

Près de 10 ans après l'accident de Fukushima, on note cependant des nuances, voire des désaccords au sein des autorités gouvernementales. Certains acteurs reconnaissent des erreurs dans la gestion post-accidentelle et entament une analyse plus réflexive sur la politique menée, le rôle des scientifiques et des experts dans les décisions prises, l'impact de ces décisions sur le public et sur les conséquences de la catastrophe pour la démocratie Japonaise. Un haut responsable gouvernemental va jusqu'à évoquer une comparaison avec la catastrophe de Minamata pour déplorer les erreurs commises pendant la gestion post accidentelle de Fukushima (Fassert et Hasegawa, 2019).

\subsection{Une lecture en termes de trustworthiness (capacité à mériter la confiance des citoyens)}

Chaque catastrophe nucléaire ravive la mémoire d'autres accidents, comme Wynne l'a montré avec Sellafield, comme on le voit à l'heure actuelle au Japon : les problèmes de gestion post-accidentelle à Fukushima ravivent la mémoire de ce qui s'est joué lors de l'accident de Tchernobyl, et au Japon, l'accident a ravivé la mémoire et les questions liées aux bombardements d'Hiroshima et de Nagasaki. Les mécanismes de confiance et de défiance au moment d'un accident héritent de ces histoires, les réactualisent. La perte de confiance liée à un accident nucléaire s'inscrit dans un imaginaire collectif et ceci de façon durable. Ainsi, le «mensonge d'Etat» (Kalmbach, 2014) lié à l'accident de Tchernobyl a laissé une trace durable en France, qui est réapparue au moment de l'accident de Fukushima. Dans la période qui a suivi l'accident, des experts de la cellule sanitaire du centre de crise de l'IRSN ont répondu aux questions du public. Ils ont parfois été confrontés, à la fin d'un entretien téléphonique 
portant sur les dangers de la situation au Japon ou en France, à la remarque suivante: "Qu'est-ce qui me prouve que vous ne mentez pas, comme vous nous avez menti en 1986 pour Tchernobyl? » (Fassert, 2013).

Une seconde dimension concerne le caractère complexe de la trustworthiness des personnes et des institutions, et son rôle dans les mécanismes de perte ou de construction de la confiance. La trustworthiness est pour Luhman une combinaison de «compétences » et de prise en compte des intérêts et de la vulnérabilité d'autrui. C'est cette vulnérabilité qui déclenche, selon Luhman, le geste de confiance : «Pour celui qui accorde sa confiance, sa vulnérabilité est l'instrument par lequel il met en branle une relation de confiance. Ce n'est qu'à partir de sa propre confiance qu'il a la possibilité de formuler à titre de norme le fait que sa confiance ne soit pas déçue et ainsi d'amener l'autre dans cette voie qu'il vient de tracer».

La dénonciation, souvent très émotionnelle, de la politique gouvernementale, se comprend très bien si on appréhende cette dimension essentielle de la vulnérabilité, et particulièrement de la reconnaissance de la vulnérabilité des plus faibles et des plus impuissants: les enfants. Il s'agit d'une dénonciation qui en appelle à l'éthique, comme on le voit dans les discours de la contestation présentés précédemment.

Quant à la dimension « compétence » de la trustworthiness, si elle est présente, il ne s'agit pas tant de considérer, pour ceux qui ont perdu confiance, que les autorités et leurs experts sont strictement incompétents. Le reproche porte davantage (d'après nos données recueillies en entretien auprès des habitants) sur le fait que ces institutions ne reconnaissent pas l'incertitude des connaissances sur les faibles doses et leurs effets, et qu'elles s'attachent au contraire à affirmer l'absence de dangerosité. Retenir l'hypothèse la plus «optimiste» ou la moins «précautionneuse» (pas de risque en dessous de $100 \mathrm{mSv}$ ) n'est pas dénoncé comme une forme d'incompétence (ce qui reviendrait à penser que ces experts ne savent pas) mais comme un choix délibéré de ne retenir, dans une situation incertaine et controversée, que les éléments, et la position, les plus à même de servir d'autres intérêts. La question des objectifs mêmes de la radioprotection peut être reposée à la lumière des travaux de Boudia (2008). En 1955, une commission chargée d'étudier les problèmes de protection que posent les rayons $\mathrm{X}$ et la radioactivité des éléments naturels ou artificiels est instituée en France. Elle doit permettre, sur la base de nouvelles préconisations de la CIPR, récemment créée, "de déterminer les doses de rayonnement permissibles pour les travailleurs et la création d'un nouveau type de norme, celui de la dose maxima pour la population en vue de répondre aux inquiétudes et controverses sur les dangers collectifs des retombées radioactives des essais atomiques $»$. Le consensus scientifique est difficile à trouver. Boudia rappelle les propos de L. Bugnard qui formulait clairement en 1958 les termes du problème auquel était confrontée la commission: "C'est un problème analogue devant lequel s'est trouvé la CIPR quand on a dî fixer les normes. Il faut aboutir à des choses raisonnables d'une part, protéger les populations, d'autre part, ne pas gêner le développement de l'énergie atomique» (Boudia, 2008). Pour Boudia, la tension, ainsi formulée, entre la protection des populations et la poursuite du développement de l'industrie nucléaire traverse l'ensemble des systèmes d'expertise de cette époque.
Face à ce type de position, l'émergence d'une expertise associative porteuse de visions scientifiques différentes est apparue plus «trustworthy», car faisant place implicitement à une forme de principe de précaution dans un contexte d'incertitudes, ou bien à la prise en compte des recherches reconnaissant la dangerosité des faibles doses. La principale caractéristique de ces contre experts, derrière leur diversité, est qu'ils ne sont jamais apparus comme visant d'abord à rassurer sur le risque radiologique. Pour certains citoyens, les contre experts ont été considérés comme les seuls acteurs prenant en compte leurs intérêts - et au-delà leur vulnérabilité - dans une situation de risque (une caractéristique majeure de la confiance selon Luhmann (2006) est le lien entre trust et risque) : c'est le cas des associations attachées avant tout à "protéger les enfants » comme l'indique le nom adopté par beaucoup d'entre elles, et le nom du réseau auquel se sont rattachés de nombreuses associations nées dans l'après Fukushima.

\section{Le processus de levée d'ordre d'évacuation}

\subsection{De nouveau un seuil de $20 \mathrm{mSv} .$.}

La question du seuil d'exposition est à nouveau posée lorsque le gouvernement souhaite lever les ordres d'évacuation dans les territoires pour lesquels ils ont été promulgués. L'accident nucléaire est déclaré «terminé » le 16 décembre 2011. Fin décembre 2011, les zones sont redéfinies; les territoires dans lesquels la situation radiologique est inférieure à $20 \mathrm{mSv} / \mathrm{an}$ deviennent des «zones prêtes pour la levée de l'ordre d'évacuation »; les territoires où la situation radiologique est supérieure à $20 \mathrm{mSv} / \mathrm{an}$ deviennent des «zones d'habitation limitée ». Parmi celles-ci, les territoires dépassant $50 \mathrm{mSv} / \mathrm{an}$ sont déclarés : «zones difficiles pour le retour». En mars 2012, le gouvernement se concerte avec la préfecture de Fukushima et les municipalités afin d'organiser les processus de Levée des Ordres d'Evacuation. La NRA constitue un comité d'experts qui réfléchit sur les éléments qui faciliteraient le retour des habitants, et établit des «Directives pour le retour» en novembre 2013. Le cadre de référence de cette politique reste le cadre fixé par la CIPR. Le terme de « situation existante » est choisi par opposition à la notion de «situation d'urgence » au moment de la crise liée à un accident. Le cadre réglementaire reste très souple dans la publication 111 , qui stipule: «les autorités nationales peuvent prendre en compte les circonstances et aussi profiter de l'agenda du programme de réhabilitation pour adopter des valeurs de référence intermédiaires qui conduisent à une amélioration progressive de la situation ».

\subsection{De nouveau, la contestation du seuil...}

Le gouvernement agit donc dans ce cadre réglementaire, en jouant notamment sur la souplesse caractérisant le passage de « la situation d'urgence» à «la situation existante» (postaccidentelle), termes retenus par la recommandation de la CIPR.

Certaines critiques vont porter sur le fait d'avoir choisi le seuil le plus élevé de la fourchette proposée par la CIPR. Ainsi, le Pr Yamauchi rappelle : «La fin de la crise a été déclarée en 
décembre 2011 par le Premier Ministre en fonction, M. Noda, qui déclare: "l'accident est terminé". Selon la CIPR, on entre désormais dans la phase de "situation existante", où on doit choisir entre 1 et $20 \mathrm{mSv}$, mais le gouvernement continue à dire qu'il a choisi la fourchette basse des 20 à $100 \mathrm{mSv}$. Ils sont donc incohérents »! (Fassert et Hasegawa, 2019)

Mais la majorité des griefs viennent surtout du fait que les travaux de décontamination des territoires ont été au départ entamés avec un objectif de retour aux « $1 \mathrm{mSv} »$ pour le retour des populations. A l'Agence pour la Reconstruction, on reconnaît que l'objectif «à long terme» reste bien de revenir aux $1 \mathrm{mSv} / \mathrm{an}$, mais on reconnaît que ce «à long terme » n'a pas été défini, ce qui sera source de critiques et de frustrations pour les habitants, qui avaient pu imaginer que les opérations de décontamination leur rendraient un territoire «propre» rapidement.

D'autres critiques portent, au-delà du seuil lui-même, sur la non-reconnaissance, par les autorités, des incertitudes liées à la décroissance radioactive des radionucléides. Ainsi le rapport ACRO réalisé pour Global chance explique: «Certains radioéléments comme le césium décroissent lentement. Le débit de dose moyen n'a diminué que de $40 \%$ en moyenne la première année au Japon (Boilley, 2015). Les travaux de décontamination n'ont pas permis d'atteindre les " $1 \mathrm{mSv}$ " qui avaient été fixés au départ comme référence pour le retour des populations ».

La première ville levant son ordre d'évacuation est Tamura, en avril 2014, suivie par le village de Kawauchi en octobre 2014, et enfin la ville de Naraha en septembre 2015. Les nouvelles directives gouvernementales établies en juin 2015 visent clairement à accélérer le «retour», la «reconstruction» dans la préfecture de Fukushima: tous les ordres d'évacuation dans les «points spécifiques» doivent être levés avant mars 2017. Elles sont assorties d'un discours insistant: «On n'abandonnera pas Fukushima», «Pas de reconstruction du Japon sans reconstruction de Fukushima». Seules les «zones de retour difficile» restent en dehors de ces directives. De plus, les autorités insistent sur le fait que les évacuations et les conditions de vie des évacués dans les lieux d'hébergement temporaire sont également à l'origine d'effets non négligeables sur la santé. Pour cela, le seuil de $20 \mathrm{mSv} / \mathrm{an}$ a été choisi, en insistant sur la nécessité, pour les habitants, de surveiller leur dosimétrie en portant un dosimètre individuel.

Cette politique fera à son tour l'objet de contestations, qui ne seront pas abordées en détail ici. On peut toutefois souligner que les $1 \mathrm{mSv} /$ an prennent un nouveau sens: celui d'une «promesse non tenue» par le gouvernement sur la possibilité d'effacer les traces de l'accident. L'enjeu symbolique, un retour à la normale possible après l'accident, et objectivé par ce seuil, est majeur. L'ACRO choisit pour titre «Retour à l'anormal» pour son rapport sur les conséquences de l'accident, à l'anniversaire des 5 ans de la catastrophe nucléaire.

La question du seuil se complexifie. D'abord, au niveau radiologique, avec l'introduction de la notion de «vraie dose » (celle indiquée par les dosimètres individuels portés par les habitants) qui s'avère inférieure aux prévisions faites sur la base de mesures effectuées dans l'environnement lors du zonage. Les autorités japonaises vont largement communiquer sur ce point, jugeant la situation rassurante, certes, mais qui résulte en partie de l'intégration dans la vie quotidienne par les personnes d'auto-contraintes pour être moins exposées (passer le moins de temps possible dehors par exemple) (Ferrier, 2011). En conséquence, cette situation en apparence rassurante n'a pas éteint la polémique sur les niveaux d'exposition réelle de la population lors d'une vie normale.

Au-delà du seuil, d'autres contestations vont surgir ou resurgir : la décontamination des zones rurales, si imparfaite, a entraîné des dépenses colossales, et plusieurs habitants pensent que l'argent dépensé aurait pu être proposé pour permettre à ceux qui le souhaitent de rebâtir une maison ailleurs.

\section{Conclusion}

Le seuil choisi pour le zonage des territoires contaminés par le gouvernement japonais et sa contestation multiforme dans l'espace public apparaîssent bien comme un objet d'étude qui cristallise de nombreuses questions relevant de la sociologie des sciences et des techniques: il montre l'asymétrie de pouvoir des différents acteurs (Frickel, 2015), et l'analyse de sa contestation montre l'apparition de questions éthiques et politiques liées à la régulation des risques, questions qui ne sont bien sûr pas spécifiques des risques liées aux radiations ionisantes.

Malgré une contestation multiforme (Lochard et al., 2019, Schneider et al., 2019), la politique du gouvernement japonais restera adossée à un seuil très contesté, y compris lors de la Levée des Ordres d'Evacuation à partir de 2014. Cependant, l'accident de Fukushima a eu pour importante conséquence de rouvrir la controverse sur les faibles doses. Cette controverse fait partie des controverses «à rebonds » comme le soulignait Boudia: «Ces controverses multiples sont à chaque fois redéfinies, reformulées et investies d'enjeux différents. Elles restent encore latentes, pouvant connaître un nouveau développement à la faveur de tel ou tel événement. Elles mettent en jeu un ensemble de valeurs, morales et politiques qui, même mouvantes, peuvent trouver à nouveau à s'exprimer sur les scènes publiques. Et à chaque fois, elles reformulent la question de la portée sociale de données scientifiques, et celle des choix technoscientifiques et des processus de décision dans les sociétés démocratiques» (Boudia, 2007).

Cependant, des arguments plus radicaux, tels que ceux portés par le Rapporteur Spécial des Nations Unies mettant en question les fondements mêmes de la radioprotection ne seront pas repris durablement par les associatifs, et la contestation continuera à s'appuyer essentiellement sur des arguments scientifiques et un appel à l'éthique, sans que la question posée par Anand Grover soit mise à l'agenda dans l'espace public.

\section{Remerciements}

Remerciements à David Boilley (ACRO) et Reiko Hasegawa (Sciences Po) pour la relecture de cet article.

\section{Références}

BEIR VII. 2006. The 2006 National Academy of Sciences Biologic Effects of Ionizing Radiation (BEIR) VII Report.

Blix H. 2005. Cited in "Chernobyl: Looking back to go forward", in : Proceedings of an International IAEA Conference, Vienna 6-7 September 2005. 
Boilley D. 2015. L'impact sanitaire de la catastrophe de Fukushima. Les cahiers de Global Chance $\mathrm{n}^{\circ} 37$.

Boilley D. 2016. Fukushima 5 ans après, retour à l'anormal. Rapport de l'Association pour le Contrôle de la Radioactivité dans l'Ouest (ACRO). Sortir du nucléaire 68: 8-9.

Boilley D, Josset M. 2016. Plan d'urgence nucléaire en France, forces et faiblesses. Rapport ACRO réalisé à la demande de l'ANCCLI.

Boudia S. 2007. Naissance, extinction et rebonds d'une controverse scientifique. Les dangers de la radioactivité pendant la guerre froide, Mil neuf cent. Revue d'histoire intellectuelle 1/2007 25: $157-170$

Boudia S. 2008. «Sur les dynamiques de constitution des systèmes d'expertise scientifique: le cas des rayonnements ionisants », Genèses 70: 26-44.

Boudia S, Jas N. 2014. Powerless science? Science and politics in a toxic world. New York: Berghahn.

CCNE. 2015. The state of affairs and ongoing challenges of the Fukushima nuclear disaster-A civil society response towards recovery. Tokyo, Japan: Citizen's Commission on Nuclear Energy.

Courdy K. 2013. Au-delà du nuage Yonaoshi 3.11. Film documentaire.

Fassert C. 2013. Des experts face à la crise. La cellule sanitaire du CTC de l'accident de Fukushima. Rapport interne IRSN.

Fassert C, Hasegawa R. 2019. Shinrai research Project: The 3/11 accident and its social consequences. Case studies from Fukushima prefecture. Rapport IRSN/2019/00178.

Ferrier M. 2011. Fukushima, récit d'un désastre. Gallimard.

Frickel S. 2015. The New Political sociology of science. University of Wisconsin.

Gaulène M. 2016. Le nucléaire en Asie. Fukushima, et après ? (P. Picquier, ed.).

Greenpeace. 2017. "Unequal impact”. Kendra Ulrich, (Ai Kashiwagi and Kazue Suzuki, Greenpeace Japan, eds.).

Grover A. 2013. Report of the Special Rapporteur on the right of everyone to the enjoyment of the highest attainable standard of physical and mental health, Mission to Japan (15-16 November 2012), A/HRC/23/41/Add.3, 2 United Nations.

Hasegawa R. 2015. Returning home after Fukushima: Displacement from a nuclear disaster and international guidelines for internally displaced persons. Migration, Environment and Climate Change: Policy Brief Series, Issue 4, Vol. 1, International Organization for Migration (IOM).

IAEA. 2009. Severe accident management programmes for nuclear power plants. Safety Guide. IAEA Safety Standards Series ${ }^{\circ}$ NSG-2.15. Vienna: International Atomic Energy Agency.

ICRP. 2009. Publication 111: Application of the Commission's recommendations to the protection of people living in long-term contaminated areas after a nuclear accident or a radiation emergency, Vol. 39, No. 3.

IPPNW/PSR. 2016. 5 years living with Fukushima. Summary of the health effects of the nuclear catastrophe. International Physicians for the Prevention of Nuclear War and Physicians for Social Responsibility report.

Jobin P. 2016. "Nuclear Gypsies" in Fukushima before and after 3/11. Forthcoming in Nuclear Portraits: People, Communities and the
Environment, Laurel MacDowell, University of Toronto Press.

Kalmbach K. 2014. Meanings of a Disaster: The Contested 'Truth' about Chernobyl. British and French Chernobyl Debates and the Transnationality of Arguments and Actors. Dissertation. Florence: European University Institute.

Lochard J, Schneider T, Ando R, Niwa O, Clement C, Lecomte JF, Tada JI. 2019. An overview of the dialogue meetings initiated by ICRP in Japan after the Fukushima accident. Radioprotection 54 (2): 87-101. https://doi.org/10.1051/radiopro/2019021.

Luhmann N. 2006. La confiance, un mécanisme de réduction de la complexité sociale. Paris : Economica, Études sociologiques (Coll.).

NAIIC. 2012. Report of the Fukushima Nuclear Accident Independent Investigation Commission of the National Diet of Japan. http://www.nirs.org/fukushima/naiic-report.pdf.

Quéré L. 2006. Confiance et engagement, in Albert Ogien et Louis Quéré (dir.) Les moments de la confiance. Connaissance, affects, et engagements. Paris : Economica, Études sociologiques (Coll.).

Rosanvallon P. 2008. La légitimité démocratique. Impartialité, réflexivité, proximité. Paris : Seuil.

Schneider T, Maitre M, Lochard L, Charron S, Lecomte JF, Ando R, Kanai Y, Kurihara M, Kuroda Y, Miyazaki M, Naito W, Orita M, Takamura N, Tanigawa K, Tsubokura M, Yasutaka T. 2019. The role of radiological protection experts in stakeholder involvement in the recovery phase of post-nuclear accident situations: Some lessons from the Fukushima-Daïchi NPP accident. Radioprotection 54(4): 259-270. https://doi.org/10.1051/radiopro/2019038.

Shirabe M, Fassert C, Hasegawa R. 2015. "Risk Communication" to Participatory Radiation Risk Assessment'. Workshop "Understanding and Communicating Risks Post-Fukushima", organisé par the United Nations University Institute for the Advanced Study of Sustainability (UNU-IAS), Fukushima Global Communication (FGC). http://i.unu.edu/media/ias.unu.edu-en/news/12850/FGCWO-21.

Sugita K, Augendre M. 2012. Les déplacés de l'accident de Fukushima: les conséquences sociales et sanitaires, et les initiatives citoyennes. HAL halshs_00967033.

Topçu S. 2014. Organiser l'irresponsabilité? La gestion (inter) nationale des dégâts d'un accident nucléaire comme régime discursif. Ecologie et politique 49: 95-114.

Topçu S. 2016. Catastrophes nucléaires et «normalisation » des zones contaminées. Enjeux politiques, économiques, sanitaires, démocratiques et éthiques. Les notes de la fondation d'Ecologie Politique 8.

USAEC. 1957. Theoretical possibilities and consequences of major accidents in large nuclear power plants. US Atomic Energy Commission Report.

Wynne B. 1992. Misunderstood misunderstandings, social identities and public uptake of science. Public Underst Sci 1(3): 281304.

Yuasa M. 2013. Whistle in the graveyard: safety discourse and Hiroshima/Nagasaki Authority in Post Fukushima Japan. in Hiroshima Shiritsu Daigaku, Kokusai Gakubu (éd.), Japan's 3/11 disaster as seen from Hiroshima: A multidisciplinary approach. Tokyo : Soeisha/Shoten.

Citation de l'article : Fassert C. 2020. L'accident nucléaire de Fukushima: enjeux et contestations autour de la définition du zonage. Radioprotection 55(1): 29-38 